EPSC Abstracts

Vol. 15, EPSC2021-335, 2021, updated on 29 Nov 2021

https://doi.org/10.5194/epsc2021-335

Europlanet Science Congress 2021

(C) Author(s) 2021. This work is distributed under

the Creative Commons Attribution 4.0 License.

\title{
OpenPlanetary, an "umbrella" non-profit organisation for open planetary science communities
}

\author{
Nicolas Manaud ${ }^{1}$, Chase Million ${ }^{2}$, Angelo Pio Rossi ${ }^{3}$, Jérôme Gasperi ${ }^{4}$, Michael Aye ${ }^{5}$, Matt Brealey ${ }^{6}$, \\ Mario D'Amore ${ }^{7}$, Alessandro Frigeri ${ }^{8}$, Trent Hare ${ }^{9}$, Emily Lakdawalla ${ }^{10}$, Emily Law ${ }^{11}$, Andrea Nass ${ }^{7}$, \\ and Mark Wieczorek ${ }^{12}$ \\ ${ }^{1}$ SpaceFrog Design, Toulouse, France (nicolas@spacefrog.design) \\ ${ }^{2}$ Million Concepts LLC, Louisville, KY, USA \\ ${ }^{3}$ Jacobs University Bremen, Department of Physics and Earth Sciences, Bremen, Germany \\ ${ }^{4}$ SnapPlanet, Toulouse, France \\ ${ }^{5}$ University of Colorado, Boulder, USA \\ ${ }^{6}$ Matt Brealey, UK \\ ${ }^{7}$ German Aerospace Centre (DLR), Institute of Planetary Research, Berlin, Germany \\ ${ }^{8}$ Istituto di Astrofisica e Planetologia Spaziali (IAPS), Istituto Nazionale di Astrofisica (INAF), Rome, Italy \\ ${ }^{9}$ U. S. Geological Survey, Astrogeology Science Center, Flagstaff, AZ, USA \\ ${ }^{10}$ Lakdawalla Group LLC, USA \\ ${ }^{11}$ Jet Propulsion Laboratory, California Institute of Technology, Pasadena, CA \\ ${ }^{12}$ Observatoire de la Côte d'Azur, Nice, France
}

Introduction: OpenPlanetary, or simply "OP", is an international non-profit organisation that promotes open research in the planetary science and exploration communities: sharing ideas and collaborating on planetary research and data analysis problems, new challenges, and opportunities [1].

OpenPlanetary started in 2015 as a way for participants of the ESA's Planetary GIS Workshop to stay connected and exchange information related to and beyond this workshop. It expanded further by playing a similar role for the second USGS-hosted Planetary Data Workshop (PDW) in 2017. OpenPlanetary has continued to support the biannual PDW and provides a more persistent forum for participants to highlight presented topics and discussions from the workshops.

In 2018, we established OpenPlanetary as a non-profit organisation (Association under 1901 French Law, [2]) in order to provide us with a legal framework to sustainably fund our community framework, projects and activities, and to better serve the planetary science community as a whole. OpenPlanetary is governed by a Board of Directors, elected for two years, which (1) define the policy and general orientation, (2) initiate, endorse, lead, or contribute to the projects and activities, and (3) can make use of the funds of the Association for any endorsed project or activity; the Bureau contains a 3-person subset of the Board members (a president, treasurer, and secretary) and serves as the executive body of the Association.

Mission: Our mission is to promote and facilitate the open practice of planetary science and data analysis for professionals and amateurs. We do so by organizing events and conducting collaborative projects aimed at creating scientific, technical and educational resources, tools and data accessible 
to all.

Members and Membership: With currently 300+ members across the world, OpenPlanetary membership is free and open to research and education professionals: scientists, engineers, designers, teachers and students, space enthusiasts and citizen scientists [3]. Although the early membership had a strong representation in planetary surface and mapping sciences, OpenPlanetary has expanded and is intended to serve as an "umbrella" for all communities of planetary data and tool users, producers or providers across scientific disciplines, space missions or working groups.

Collaboration Platform: Our collaboration platform mainly consists of fully-featured Slack and Github instances. OP members use OP Slack workspace to stay connected and have real-time discussions with other members [4], and are entitled to request admin rights to host and manage open source projects on OP Github organization [5].

Online Forum: We provide a public online OP Forum for research professionals and amateurs across all planetary science disciplines and communities to find help, share and discuss data, tools and resources [6]. While OP Slack is considered for the more informal discussions, the OP Forum is intended to post Q/A and discussion "gems" from OP Slack, or any resources that would help a broader community (eg: a short tip, a handy how-to guide or a list of curated resources), and that would benefit from having a permanent web-presence and being discoverable by search engines.

Data Cafés: Since 2017, we have organised Data Cafés at scientific conferences for people to meet, share, discuss and solve common challenges and issues related to planetary data handling and analysis. These events follow an "unconference" format allowing and encouraging anyone to propose a topic and lead a group activity (eg: demo, tutorial, hack), or simply to ask for help [7].

Online Events: Unable to continue with the in-person Data Cafés in 2020, we started hosting virtual online events: (1) OPvCon in June 2020 was our first free virtual conference, scheduled in place of the cancelled Planetary Science Informatics and Data Analytics Conference (PSIDA). It consisted of lecture-length talks from invited speakers, networking opportunities, workshops and tutorials, and a hackathon [8], and (2) since March 2020, we have hosted weekly OP Lunch Talks to present and discuss technical topics of interest to the planetary science community [9]. Most of these events are recorded and made publicly available on YouTube. They now represent a substantial collection of high-quality informational resources and training videos on diverse topics related to planetary science [10].

Community Projects: Our flagship project is OpenPlanetaryMap (OPM), an open planetary mapping and social platform and effort to foster planetary mapping and cartography on the web for all [11]. We also support PlanetaryPy, a community effort to develop a core package for planetary science in Python and foster interoperability between Python planetary science packages [12]. A number of other projects not strictly homed under the OP umbrella have arisen from collaborations fostered in OP Slack or during OP Lunch discussion sessions.

Outlook: We held our first yearly OpenPlanetary General Assembly in December 2020 [13], during which a new Board of Directors was elected. Our main focus within the next couple of years is on (1) consolidating and expanding OP Lunch and other virtual activities, (2) increasing the usage and impact of the OP Forum for all communities (eg: Planetary Spatial Data Infrastructures (SDI) communities), and (3) identifying sustainable funding opportunities.

References: [1] https://www.openplanetary.org, [2] https://www.journalofficiel.gouv.fr/associations/detail-annonce/associations_b/20180009/457, [3] https://www.openplanetary.org/join, [4] http://openplanetary.slack.com, [5] https://github.com/openplanetary, [6] https://forum.openplanetary.org [7] https://github.com/openplanetary/op-data-cafe, [8] https://www.openplanetary.org/vcon, [9] https://www.openplanetary.org/vlunch, [10] https://www.youtube.com/openplanetary, [11] https://www.openplanetary.org/opm,

https://planetarypy.org, 
https://drive.google.com/open?id=1QfGzTT760DpTCFucCaOsOGFLY8Wyy1rE 